\title{
A Systemic Meta-Model for Socio-Environmental Systems
}

\author{
Jérôme Dantan, Yann Pollet and Salima Taibi
}

\begin{abstract}
We propose a systemic meta-model for the sustainable simulation of socio-environmental complex systems. The approach presented integrates data uncertainty management, for both representing and manipulating rigorously quantities which may have a finite number of possible or probable values with their interdependencies. We also provide an operationalization of such models for both data retrieving, via an object-relational mapping, and model simulation, via series of triples, which are linked to examples in the field of agriculture.
\end{abstract}

J. Dantan $(\bowtie) \cdot$ Y. Pollet

CEDRIC, CNAM, 292 Rue Saint-Martin, Paris 75003, France

e-mail: jdantan@esitpa.fr

Y. Pollet

e-mail: yann.pollet@cnam.fr

J. Dantan · S. Taibi

Agri'terr, Esitpa, 3 Rue Du Tronquet CS 40118, Mont-Saint-Aignan 76134, France

e-mail: staibi@esitpa.fr 